\title{
Innate immune adaptor MyD88 deficiency prevents skin inflammation in SHARPIN-deficient mice
}

\author{
Bhesh Raj Sharma $\mathbb{I}^{1} \cdot$ Rajendra Karki $^{1} \cdot$ Ein Lee ${ }^{1,2} \cdot$ Qifan Zhu $^{1,2} \cdot$ Prajwal Gurung $^{1,3} \cdot$ Thirumala-Devi Kanneganti $^{1}$
}

Received: 20 May 2018 / Revised: 18 June 2018 / Accepted: 19 June 2018 / Published online: 23 July 2018

(c) ADMC Associazione Differenziamento e Morte Cellulare 2018

\begin{abstract}
Mice deficient in SHANK-associated RH domain-interacting protein (SHARPIN), a component of the linear ubiquitin chain assembly complex (LUBAC), develop a spontaneous inflammatory disorder with pathologic hallmarks similar to atopic dermatitis and psoriasis in humans. Previous studies identified the crucial role of components of the TNF and IL-1 signaling pathways in the progression of disease in SHARPIN-deficient mice. However, an innate immune adaptor or sensor that relates to the disease progression has remained unknown. In this study, we found that the genetic ablation of myeloid differentiation primary response 88 (MyD88) completely rescued skin inflammation in SHARPIN-deficient (Sharpin ${ }^{\mathrm{cpdm}}$ ) mice. Systemic inflammation and immune cell dysregulation were partially rescued. Fibroblasts derived from Sharpin ${ }^{\mathrm{cpdm}} M y d 88^{-/-}$mice failed to provide protection against TNF-induced cell death. Sharpin ${ }^{\mathrm{cpdm}} M y d 88^{-/-}$mice had reduced TNF production in their skin. Furthermore, depletion of the microbiota through the oral administration of antibiotics (ABX) partially rescued both the skin inflammation and systemic inflammation, demonstrating a role for the gut microbiota in SHARPIN-deficient mice. Our findings suggest a detrimental role for the innate immune adaptor MyD88 in instigating skin inflammation in Sharpin ${ }^{\text {cpdm }}$ mice.
\end{abstract}

\section{Introduction}

A spontaneous single-base-pair deletion in exon 1 of the gene encoding Shank-associated RH domain-interacting protein (SHARPIN) was first identified in C57BL/Ka mice [1]. This mutation results in the complete loss of SHARPIN protein, and these mice develop a phenotype known as chronic proliferative dermatitis (CPDM), which is accompanied by multi-organ inflammation and immune system dysregulation. Consequently, they are designated

Edited by A. Villunger.

Electronic supplementary material The online version of this article (https://doi.org/10.1038/s41418-018-0159-7) contains supplementary material, which is available to authorized users.

Thirumala-Devi Kanneganti

thirumala-devi.kanneganti@stjude.org

1 Department of Immunology, St. Jude Children's Research Hospital, Memphis, TN 38105, USA

2 Integrated Biomedical Sciences Program, University of Tennessee Health Science Center, Memphis, TN 38163, USA

3 Inflammation Program, University of Iowa, Iowa City, IA, USA
$\operatorname{Sharpin}^{\mathrm{cpdm}}$ mice [2]. The dermatitis observed in these mice is similar to that seen in several human inflammatory skin diseases, such as cryopyrin-associated periodic syndrome, familial Mediterranean fever, and neutrophilic dermatoses [3]. SHARPIN, along with heme-oxidized IRP2 ubiquitin ligase 1 homolog (HOIL-1) and HOIL-1-interacting protein (HOIP), forms a ubiquitin ligase complex called the linear ubiquitin chain assembly complex (LUBAC), which generates linear ubiquitin chains to activate NF- $\mathrm{KB}$-mediated transcription [4-6]. Moreover, SHARPIN is a critical regulator of TNF-induced apoptosis and necroptosis in epidermal keratinocytes. The deletion of essential components of TNF signaling and cell-death pathways prevents the development of dermatitis in Sharpin ${ }^{\mathrm{cpdm}}$ mice [7, 8]. Furthermore, we recently determined that the inflammasomedependent cytokine IL-1 $\beta$ contributes to pathogenesis in Sharpin $^{\mathrm{cpdm}}$ mice [9]. The production of pro-inflammatory cytokines, including TNF and IL-1 $\beta$, is regulated by myeloid differentiation primary response 88 (MyD88), which is a common adaptor molecule for toll-like receptors (TLRs) and IL-1 receptors [10]. In fact, signaling via TLRs and interleukin 1 receptor (IL-1R) occurs through the sequential recruitment of MyD88 and IL-1R-associated kinase (IRAK). The signaling via MyD88 is prompted to produce 
inflammatory cytokines upon the recognition of various endogenous molecules, as well as microbial signature molecules [11]. Mice lacking MyD88 failed to produce TNF, IL-1 $\beta$, or IL-6 in response to bacterial lipopolysaccharide (LPS), demonstrating that MyD88 is a critical molecule for LPS response [12]. Given the role of MyD88 in the production of TNF and IL- $1 \beta$, and the observation that deleting essential components of TNF signaling and IL$1 \mathrm{R}$ rescued or delayed the pathogenesis in Sharpin ${ }^{\mathrm{cpdm}}$ mice, we hypothesized that a deficiency of the innate immune adaptor MyD88 might prevent skin inflammation in Sharpin ${ }^{\mathrm{cpdm}}$ mice.

\section{Results}

\section{MyD88 deficiency prevents skin inflammation in SHARPIN-deficient mice}

Initial studies have found a role for TNF and IL- $1 \beta$ signaling in instigating dermatitis in Sharpin ${ }^{\mathrm{cpdm}}$ mice [7, 9]. TLR responses for the induction of TNF and IL- $1 \beta$ and signaling mediated through IL-1R both require a common adaptor molecule, MyD88 [13]. Therefore, we investigated the role of MyD88 in the inflammatory skin phenotype in Shar-

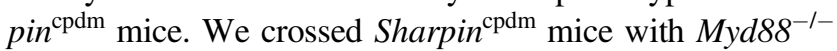
mice to generate Sharpin ${ }^{\mathrm{cpdm}} M y d 88^{-/-}$mice and scored these mice for disease progression. As expected, Sharpin ${ }^{\mathrm{cdm}}$ mice started to develop signs of dermatitis as early as postnatal day 42, whereas Sharpin ${ }^{\mathrm{cpdm}} M y d 88^{-1-}$ mice had developed no signs of the disease at 120 days (Fig. 1a, b). Next, we performed histologic analysis of skin sections from WT, Sharpin ${ }^{\mathrm{cdm}}$, and Sharpin ${ }^{\mathrm{cdm}} M y d 88^{-/-}$mice. There was epidermal thickening and increased cellular infiltration in the dermis of Sharpin ${ }^{\text {cpdm }}$ mice, as compared to that of WT mice (Fig. 1c, d). Sharpin ${ }^{\mathrm{cpdm}} M y d 88^{-/-}$mice had less cellular infiltration and less epidermal thickening when compared to Sharpin ${ }^{\text {cpdm }}$ mice. Collectively, these data show that the genetic deletion of MyD88 in Sharpin ${ }^{\mathrm{cpdm}}$ mice prevents the development of skin inflammation.

\section{MyD88 promotes systemic inflammation and immune cell dysregulation in SHARPIN-deficient mice}

In addition to developing skin inflammation, Sharpin ${ }^{\text {cpdm }}$ mice suffer from major immune cell dysregulation [9]. To further investigate whether MyD88 deletion prevented systemic inflammation, we first analyzed the size and weight of the spleens of Sharpin ${ }^{\text {cpdm }}$ mice and WT control animals (Fig. 2a, b). As expected, the spleens of Sharpin ${ }^{\mathrm{cpdm}}$ mice were enlarged relative to those of WT mice. However, the spleens of Sharpin ${ }^{\mathrm{cpdm}} M y d 88^{-/-}$mice were greatly reduced in size and weight when compared with the spleens of Sharpin ${ }^{\text {cpdm }}$ mice (Fig. 2a, b). Moreover, the number of splenocytes was also substantially lower in Sharpin ${ }^{\text {cpdm- }}$ Myd $88^{-/-}$mice (Fig. 2c). An analysis of the major immune cell populations within the spleens revealed that MyD88 deletion in Sharpin ${ }^{\text {cpdm }}$ mice significantly lowered the frequency of neutrophils (as determined by the number of Gr1 ${ }^{+} \mathrm{CD} 11 \mathrm{~b}^{+}$cells detected by flow cytometry) (Fig. $2 \mathrm{~d}$, g). However, the frequency of $\mathrm{CD} 19^{+} \mathrm{MHCII}^{+}$cells was increased (Fig. 2e, h), whereas that of $\mathrm{CD}^{+}$and $\mathrm{CD} 8^{+}$cells was unchanged in Sharpin ${ }^{\mathrm{cpdm}} M y d 88^{-1-}$ mice (Fig. 2f, i-j). We also analyzed these immune cell subsets in peripheral blood leukocytes (PBLs) and obtained similar results (Fig. 1S). These data suggest that MyD88 promotes systemic inflammation and partially regulates immune cell dysregulation in Sharpin ${ }^{\mathrm{cpdm}}$ mice.

\section{MyD88 regulates TNF production, but not TNF signaling, in the skin of SHARPIN-deficient mice}

The activation of TLRs leads to the production of MyD88dependent pro-inflammatory cytokines, including TNF [14]. To gain some insight into the role of MyD88 in promoting pathogenesis in Sharpin ${ }^{\mathrm{cpdm}}$ mice, we determined the levels of pro-inflammatory cytokines in the skin of WT, Sharpin $^{\text {cpdm }}$, and Sharpin ${ }^{\text {cpdm } M y d 88^{-l-}}$ mice. The levels of TNF, IL-1 $\beta$, and IL-6 in the skin of Sharpin ${ }^{\mathrm{cpdm}}$ mice were higher than those in the skin of WT mice (Fig. 3a). MyD88deficient Sharpin ${ }^{\mathrm{cpdm}}$ mice had a reduced level of TNF, but not of other inflammatory cytokines, suggesting that MyD88 specifically regulates TNF production in the skin of Sharpin $^{\text {cpdm }}$. We confirmed this and found decreased expression of the gene encoding TNF (Fig. 3a). SHARPIN is required for TNFR1-mediated gene induction and for the prevention of TNF-mediated death of various cells, including epidermal keratinocytes [7]. SHARPIN deficiency blunts the TNFR1 pro-survival transcriptional signal and sensitizes cells to TNF-induced cell death [7]. The skin inflammation in Sharpin ${ }^{\text {cpdm }}$ mice is characterized by epidermal cell death marked by cleaved caspase-3, -7, and -8positive cells [15]. Consistent with the literature, there was more cleavage of caspase- $3,-7$, and -8 in the skin of Sharpin $^{\mathrm{cpdm}}$ mice than that of control animals (Fig. 3b). We observed decreased cleavage of those caspases in the skin of Sharpin $^{\text {cpdm }}$ mice lacking MyD88, which might be the outcome of reduced inflammatory responses in Sharpin $^{\mathrm{cpdm}} M y d 88^{-1-}$ mice (Fig. 3b). To determine whether MyD88 regulated cell death in Sharpin ${ }^{\text {cpdm }}$ mice, we compared the cleavage of those caspases at an early timepoint (post-natal day 25) when there was no obvious difference in the development of disease (pre-diseased mice) among WT, Sharpin $^{\mathrm{cpdm}}$, and Sharpin ${ }^{\mathrm{cpdm}}$ Myd88 ${ }^{-l-}$ mice. We found less cleavage of caspase-3, -7, and -8 in Sharpin ${ }^{\mathrm{cpdm}} M y d 88^{-/-}$ 

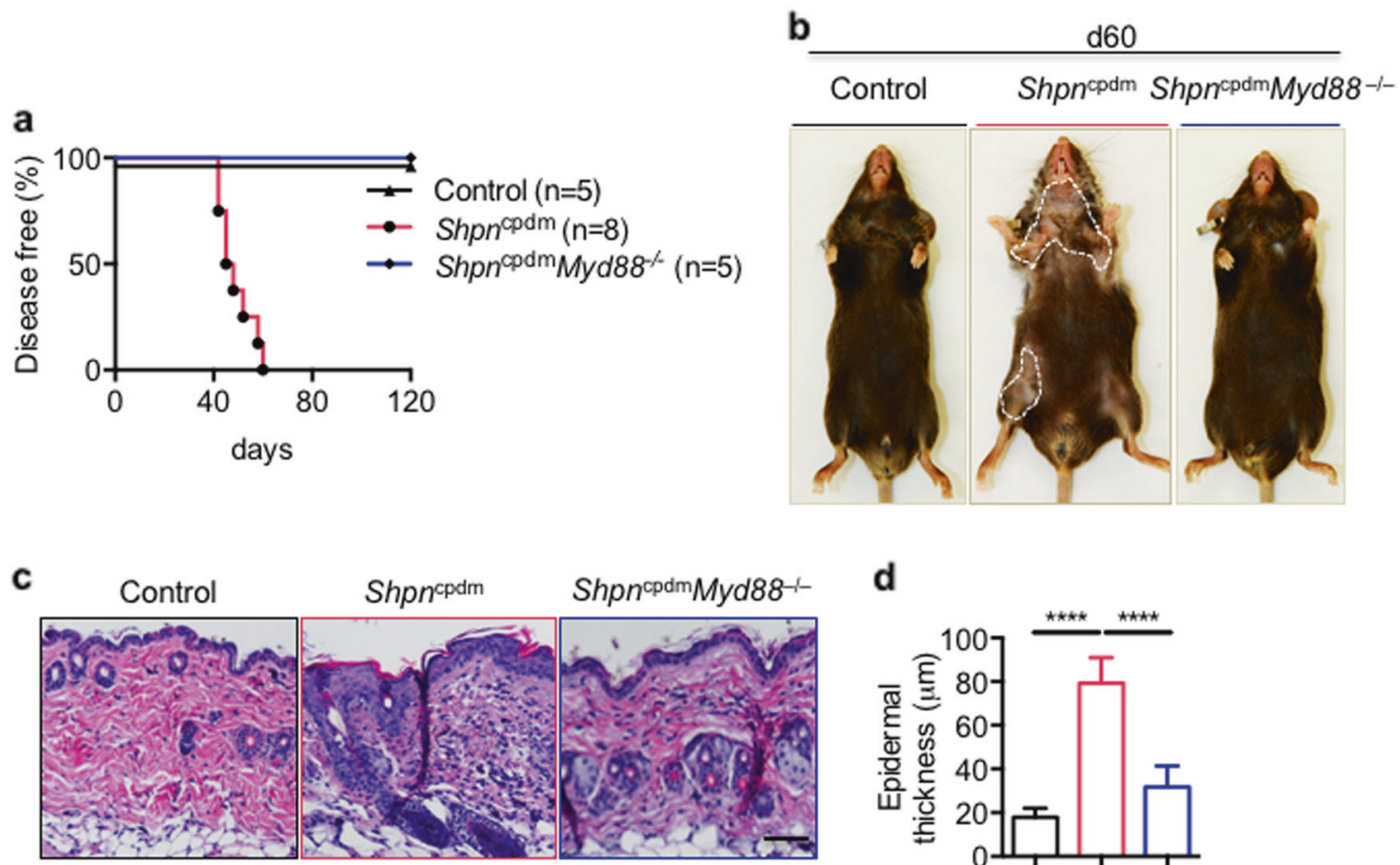

d

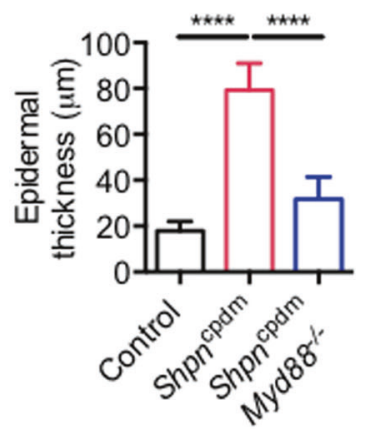

Fig. 1 MyD88 deficiency prevents skin inflammation in Sharpin ${ }^{\text {cpdm }}$ mice. a Control $(n=5)$, Sharpin ${ }^{\mathrm{cpdm}}(n=8)$, and Sharpin ${ }^{\mathrm{cpdm}} M y d 88^{-/-}$ $(n=5)$ mice were followed after weaning and scored for the onset of dermatitis. Mice that showed any sign of skin inflammation were scored as disease positive and are indicated in the disease curves on that day. The disease curve in a was analyzed by log-rank (MantelCox) testing. b Representative image of control, Sharpin ${ }^{\mathrm{cpdm}}$, and Sharpin ${ }^{\mathrm{cpdm}} M y d 88^{-1-}$ on post-natal day 60 , showing the extent of dermatitis. The white dotted lines outline the areas of dermatitis in the

mice than in Sharpin ${ }^{\text {cpdm }}$ mice, suggesting that MyD88 regulates cell death mediated by TNF in Sharpin ${ }^{\mathrm{cpdm}}$ mice (Fig. 3c). To understand the mechanistic basis of the reduced cell death in Sharpin ${ }^{\mathrm{cpdm}} M y d 88^{-1-}$ mice, we assessed cell death in response to TNF in mouse-ear fibroblasts from WT, Sharpin ${ }^{\mathrm{cpdm}}$, and Sharpin ${ }^{\mathrm{cpdm}} M y d 88^{-/-}$ mice. Consistent with the results of initial studies [7], the fibroblasts derived from Sharpin ${ }^{\mathrm{cpdm}}$ mice underwent TNFmediated apoptosis. However, the Sharpin ${ }^{\mathrm{cpdm}}$ cells lacking MyD88 were not protected against TNF-mediated cell death (Fig. 3d), suggesting that MyD88 does not regulate cell death downstream of TNF. To further confirm this, we measured TNF production and gene expression in prediseased Sharpin ${ }^{\mathrm{cpdm}}$ mice and found that the TNF levels were higher than those in WT mice. However, the TNF levels were significantly reduced in Sharpin ${ }^{\mathrm{cpdm}} M y d 88^{-/-}$ mice at a similar timepoint (Fig. 3e), suggesting that MyD88 regulates the production of TNF, but not the signaling mediated through TNFR.
Sharpin $^{\text {cpdm }}$ mouse. c Representative hematoxylin and eosin (H\&E) images of control, Sharpin ${ }^{\mathrm{cpdm}}$, and Sharpin ${ }^{\mathrm{cpdm}} M y d 88^{-/}$mice. d Epidermal thickness of skin sections from control, Sharpin ${ }^{\mathrm{cpdm}}$, and Sharpin ${ }^{\mathrm{cpdm}} M y d 88^{-1-}$ mice. Shpn ${ }^{\text {cpdm }}$ corresponds to Sharpin ${ }^{\mathrm{cpdm}}$ mice. For the H\&E sections, the epidermis was measured using FIJI ImageJ open-source software. For each section, three independent measurements were taken and averaged to obtain a measurement of the epidermis thickening. $n=5-7$ for each genotype. $P$-values less than 0.05 are considered statistically significant. $* * * * P<0.0001$

\section{Microbiota modulation partially delays skin inflammation and immune cell dysregulation in SHARPIN-deficient mice}

MyD88 signaling can be induced by endogenous molecules, extracellular components, or microbes [16]. Therefore, to examine the role of the microbiota in disease progression in Sharpin $^{\mathrm{cpdm}}$ mice, we administered a broad-spectrum antibiotic cocktail $(\mathrm{ABX})$ to these mice in their drinking water immediately after they were weaned. There was a significant delay in the development of disease in Sharpin ${ }^{\text {cpdm }}$ mice treated with antibiotics, as compared to control animals (Fig. 4a, b). Consistent with the delayed disease progression, histologic examination of the skin of Sharpin ${ }^{c p d m}$ mice treated with antibiotics showed decreased epidermal thickness and reduced cellular infiltration into the dermis, as compared to the skin of untreated Sharpin ${ }^{\mathrm{cpdm}}$ mice (Fig. 4c, d). To further investigate whether microbiota modulation rescued systemic inflammation, we analyzed the size and 
a

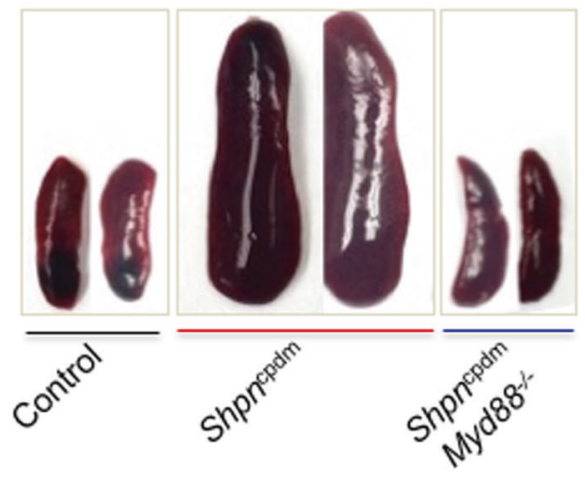

d
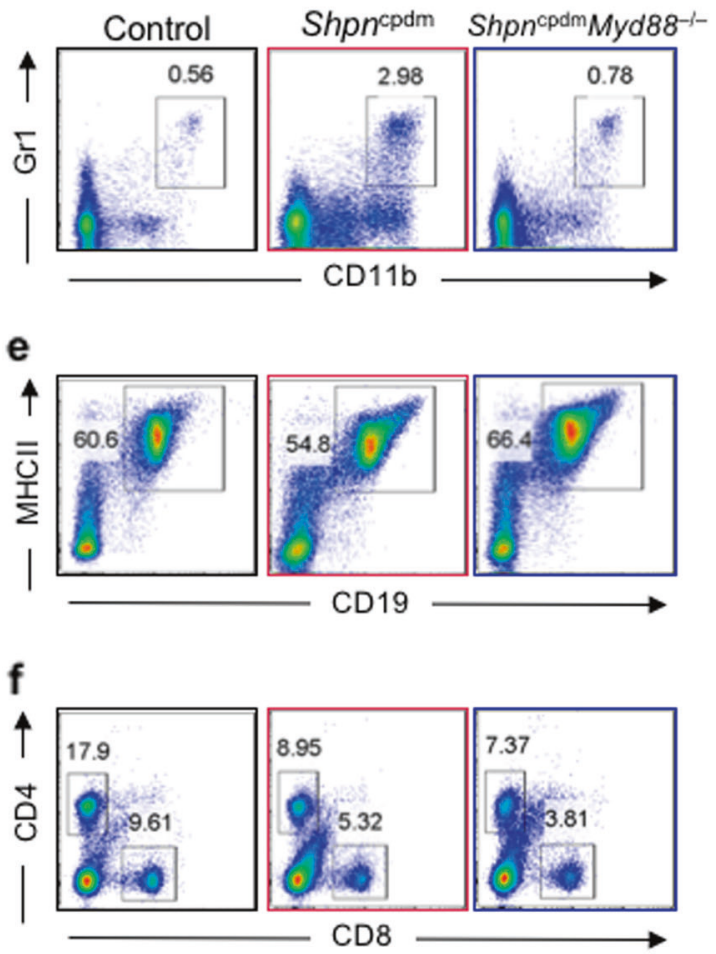

Fig. 2 MyD88 promotes systemic inflammation and immune cell dysregulation in Sharpin ${ }^{\text {cpdm }}$ mice. a Representative image of spleens of control, Sharpin ${ }^{\mathrm{cpd}}$, and Sharpin ${ }^{\mathrm{cpdm}} \mathrm{Myd} 88^{-/-}$on post-natal day 60. b, c Spleen weight (b), and splenocytes present (c) in control, Sharpin $^{\mathrm{cpdm}}$, and Sharpin ${ }^{\mathrm{cpdm}} M y d 88^{-/-}$mice on day 60. d-f Flow cytometry analysis of splenocytes from control, Sharpin ${ }^{\mathrm{cpdm}}$, and Sharpin ${ }^{\mathrm{cpdm}} \mathrm{Myd} 8^{-/-}$mice on post-natal day 60. Representative flow

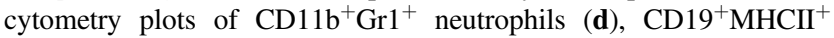

weight of the spleens. Antibiotic-treated Sharpin ${ }^{\mathrm{cpdm}}$ mice displayed significantly less splenomegaly when compared to untreated Sharpin ${ }^{\text {cpdm }}$ mice (Fig. 5a, b). Moreover, the number of splenocytes was also significantly lower in antibiotic-treated mice (Fig. 5c). Consistent with these results, microbiota modulation significantly lowered the frequency of neutrophils (as determined by the number of b

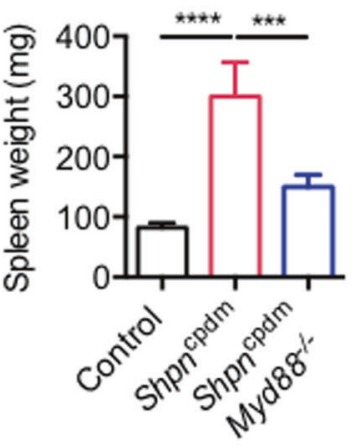

g
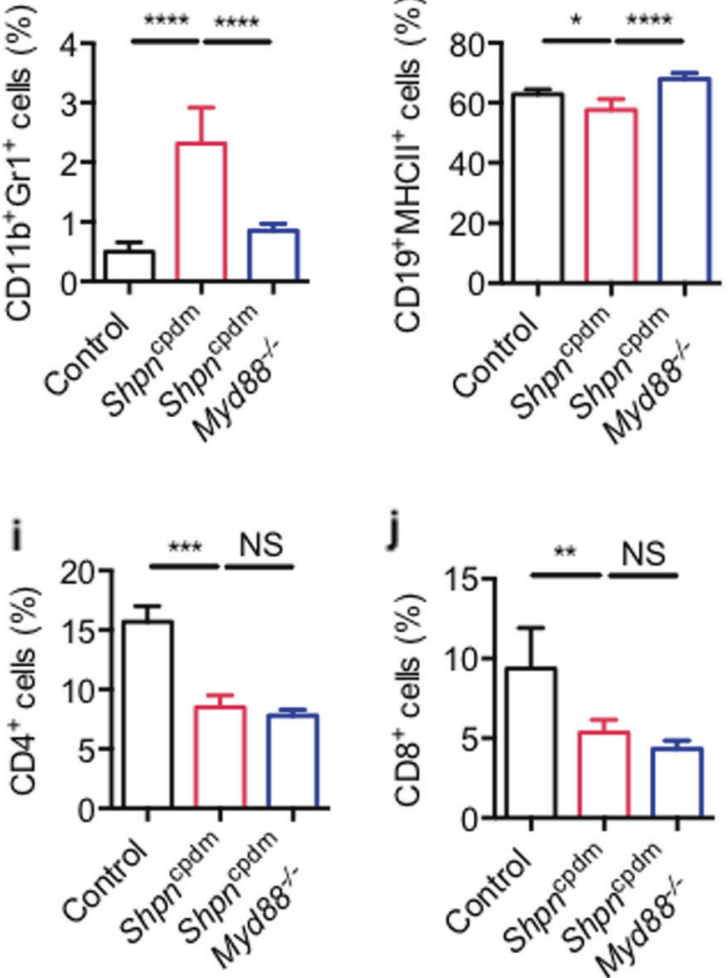

$\mathrm{B}$ cells (e), and $\mathrm{CD}^{+}$and $\mathrm{CD}^{+} \mathrm{T}$ cells (f) in the spleen. $\mathbf{g}-\mathbf{j}$ Cumulative bar charts representing the frequencies of $\mathrm{CD} 11 \mathrm{~b}^{+} \mathrm{Gr} 1^{+}$ neutrophils $(\mathbf{g}), \mathrm{CD} 19^{+} \mathrm{MHCII}^{+} \mathrm{B}$ cells $(\mathbf{h}), \mathrm{CD}^{+}{ }^{+} \mathrm{T}$ cells $(\mathbf{i})$, and CD8 ${ }^{+} \mathrm{T}$ cells $(\mathbf{j})$ in the spleen. Shpn ${ }^{\text {cpdm }}$ corresponds to Sharpin ${ }^{\text {cpdm }}$ mice. In the bar charts, data are presented as the mean \pm SEM and are representative of at least two independent experiments. $n=5-7$ for each genotype. $P$-values less than 0.05 are considered statistically significant. $* P<0.05, * * P<0.01, * * * P<0.001, * * * * P<0.0001$

$\mathrm{Gr}^{+} \mathrm{CD} 11 \mathrm{~b}^{+}$cells) (Fig. $5 \mathrm{~d}$ and g), whereas the frequency of $\mathrm{CD} 19^{+}$cells was unchanged (Fig. $5 \mathrm{e}, \mathrm{h}$ ) and the frequency of $\mathrm{CD}^{+}$and $\mathrm{CD}^{+}$cells was increased (Fig. 5f, i-j). Our analysis of PBLs yielded similar results (Fig. 2S). Overall, these results suggest that the microbiota partially regulates both the skin and systemic inflammation in Sharpin $^{\mathrm{cpdm}}$ mice. 
a

d60

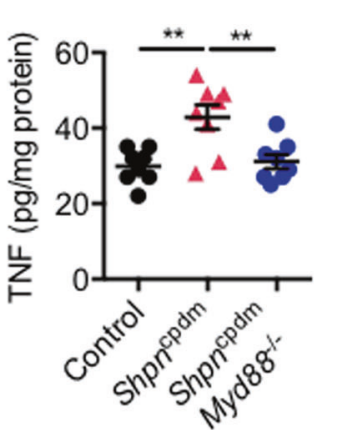

b

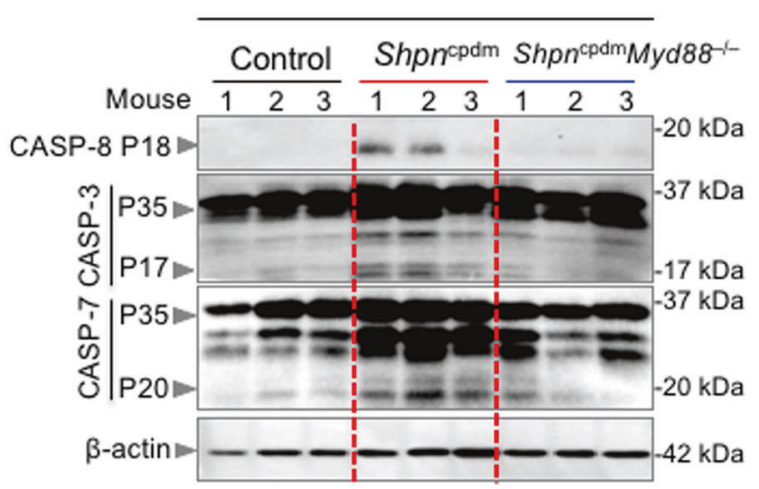

d

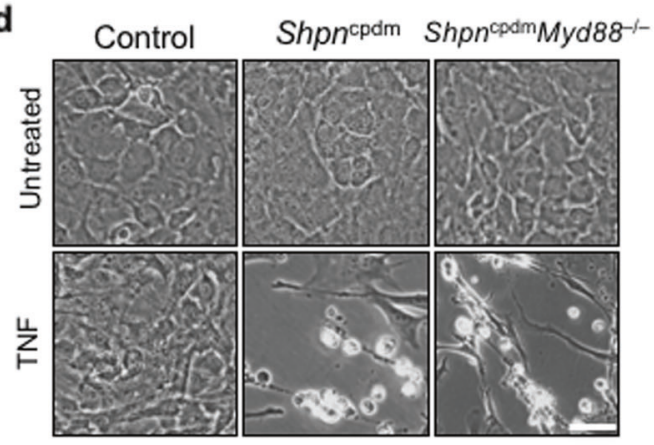

Fig. 3 MyD88 regulates TNF production, but not TNF signaling, in the skin of Sharpin ${ }^{\text {cpdm }}$ mice. a Representative bar charts of TNF, IL$1 \mathrm{~b}$, and IL-6 production and Tnf gene expression as measured in skin lysates of mice at post-natal day 60. b, c Representative Western blots of the apoptotic caspases (caspase-3, -7, and -8) from skin lysates of mice at day 60 (b) and pre-diseased mice at day 25 (c). d Fibroblasts

\section{Discussion}

SHARPIN, a component of the LUBAC, has emerged as an important regulator of diverse cellular signaling pathways, and its deficiency leads to severe dermatitis with systemic inflammation [2, 5]. TNF signaling drives apoptotic cell death in keratinocytes lacking SHARPIN [8]. The deletion of apoptosis-associated molecules downstream of the TNF

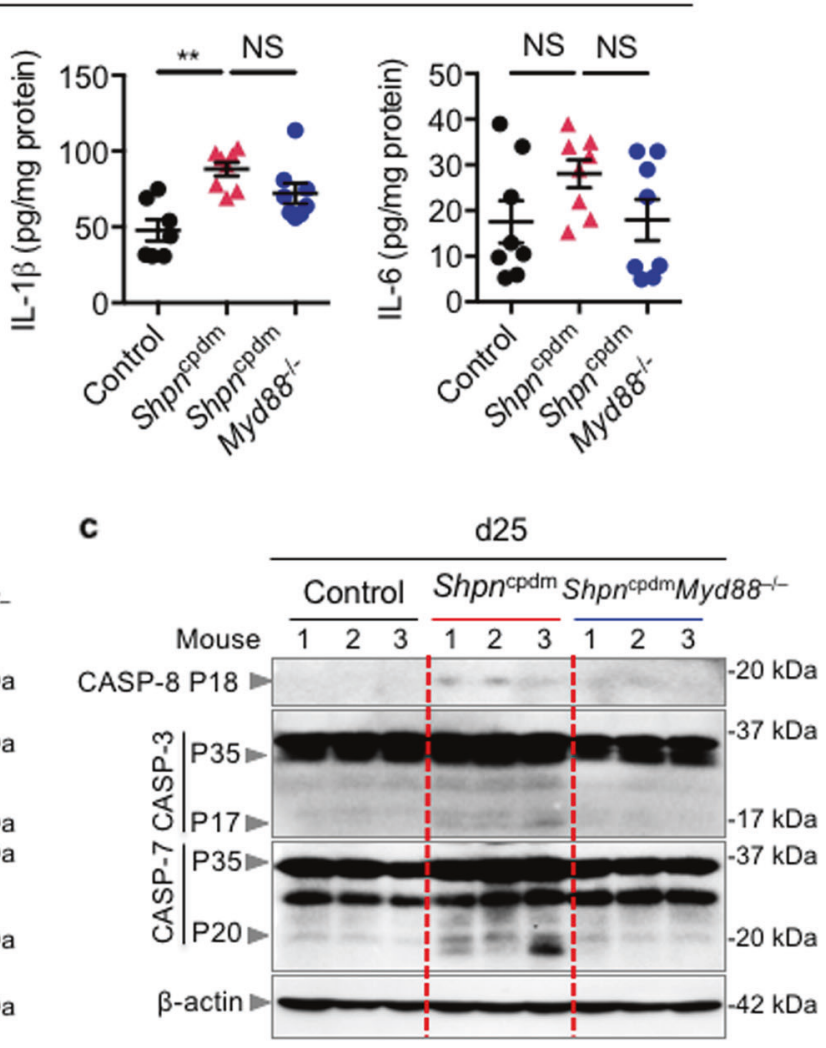

e

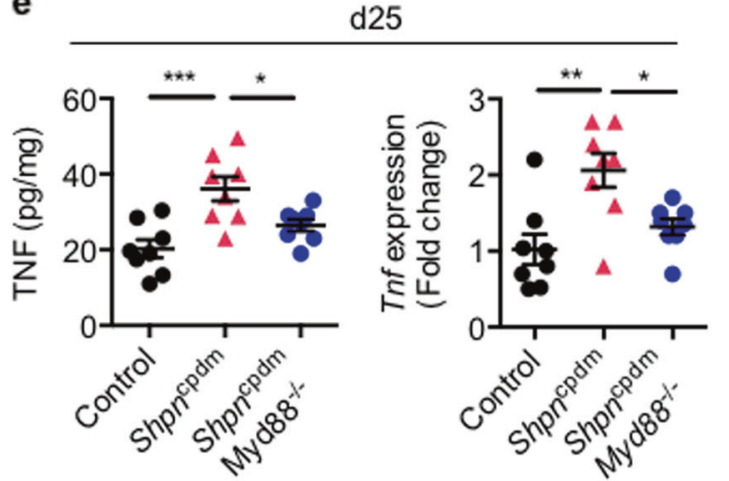

derived from control, Sharpin ${ }^{\mathrm{cpdm}}$, and Sharpin ${ }^{\mathrm{cpdm}} M y d 88^{-/-}$mice. Shpn ${ }^{\text {cpdm }}$ corresponds to Sharpin ${ }^{\text {cpdm }}$ mice. e Representative bar charts of TNF production and gene expression as measured in skin lysates of day 25 pre-diseased mice. $n=5-7$ for each genotype. $P$-values less than 0.05 are considered statistically significant. $* P<0.05, * * P<$ $0.01, * * * P<0.001$

signaling pathway, including TRADD, CASP8, FADD, and RIP1, prevents the onset of dermatitis in Sharpin ${ }^{\text {cpdm }}$ mice, suggesting that TNF-mediated cell death is critical for inducing skin inflammation in these animals [7, 8]. Although the molecules downstream of TNF signaling are well established, the roles of other molecules involved in TNF regulation have been unclear. In this study, we found that deleting MyD88, a common adaptor molecule for TLR 
b

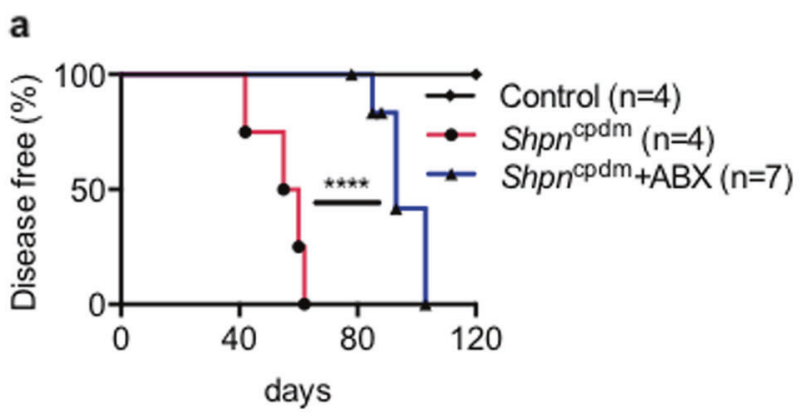

d60

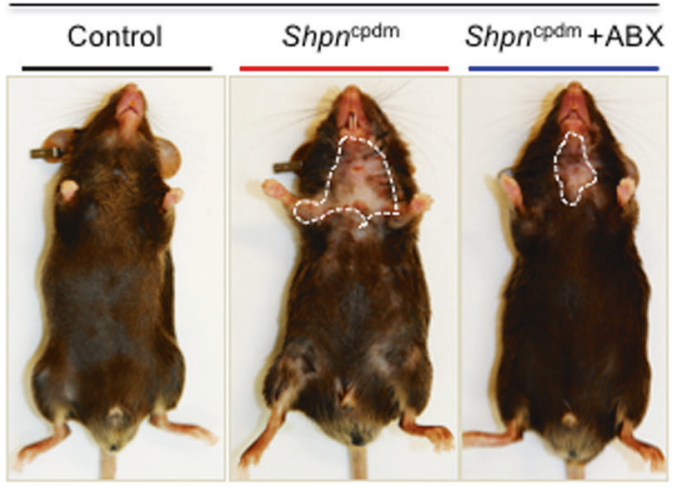

C

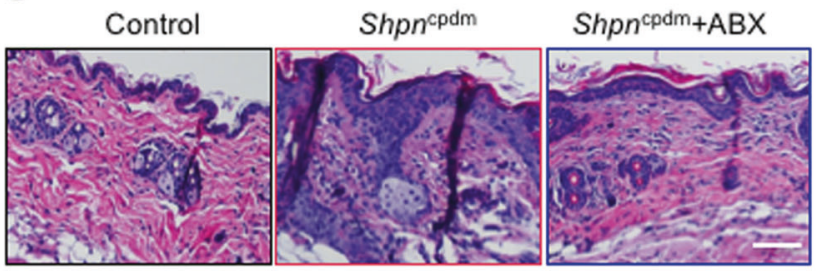

d

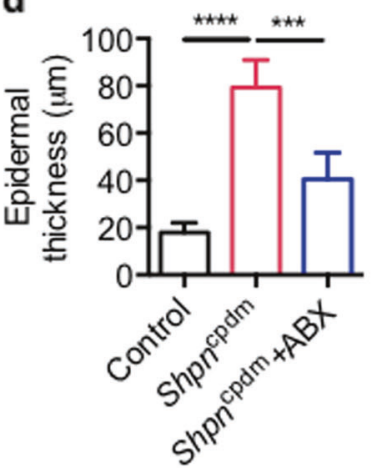

Fig. 4 Microbiota modulation partially delays skin inflammation in Sharpin $^{\text {cpdm }}$ mice. a Control $(n=4)$, Sharpin ${ }^{\text {cpdm }}(n=4)$, and Shar$p_{i n}^{\text {cpdm }}+\mathrm{ABX}(n=7)$ mice that received broad-spectrum antibiotics in their water after weaning were scored for the onset of dermatitis. Mice showing any sign of skin inflammation were scored as disease positive and are indicated in the disease curves on that day. The disease curves were analyzed by log-rank (Mantel-Cox) testing. b Representative image of control, Sharpin ${ }^{\mathrm{cpdm}}$, and Sharpin ${ }^{\mathrm{cpdm}}+\mathrm{ABX}$ mice on post-natal day 60 , showing the extent of the dermatitis, which is outlined by white dotted lines. c Representative hematoxylin and

or IL-1R signaling pathways, prevented skin inflammation in Sharpin ${ }^{\text {cpdm }}$ mice (see Fig. 6 for a schematic model). We, along with other groups, have previously demonstrated that the deletion of IL-1 $\beta$ or IL-1R delays the onset of skin disease in Sharpin ${ }^{\mathrm{cpdm}}$ mice [9]. MyD88 regulates several innate immune-signaling pathways, including microbial sensing [17], and we have shown that a deficiency in MyD88 blocks the sequence of events that leads to inflammation via the regulation of TNF production. The deletion of TNFR in epidermal keratinocytes is sufficient to prevent disease development in Sharpin ${ }^{\text {cpdm }}$ mice [8], suggesting that TNF signaling in epidermal keratinocytes drives skin inflammation in Sharpin ${ }^{\text {cpdm }}$ mice. An important question for future investigation is whether the ablation of MyD88 signaling in epidermal keratinocytes can protect Sharpin ${ }^{\text {cpdm }}$ mice from disease progression. In vivo, TNF is produced by many cell types, including myeloid, lymphoid, and stromal cells [18]. Given that the major source of TNF eosin (H\&E) images of control, Sharpin ${ }^{\mathrm{cpdm}}$, and Sharpin ${ }^{\mathrm{cpdm}}+\mathrm{ABX}$ mice. d Epidermal thickness of skin sections from control, Sharpin $^{\text {cpdm }}$, and Sharpin ${ }^{\mathrm{cpdm}}+\mathrm{ABX}$ mice. For the H\&E sections, the epidermis was measured using FIJI ImageJ open-source software. Shpn ${ }^{\mathrm{cpdm}}$ corresponds to Sharpin ${ }^{\mathrm{cpdm}}$ mice. For each section, three independent measurements were taken and averaged to obtain a measurement of the epidermis thickening. $n=5-7$ for each group. $P$ values less than 0.05 are considered statistically significant. $* * * P<$ $0.001, * * * * P<0.0001$

is myeloid cells [19], it is less likely that MyD88 functions to produce TNF in keratinocytes. TNF produced by myeloid cells might act on epidermal keratinocytes to sensitize them for apoptosis in the absence of SHARPIN. In that case, the deletion of MyD88 in myeloid cells would inhibit TNF production and skin disease in Sharpin ${ }^{\text {cpdm }}$ mice. TNFR1 signaling in non-epidermal cells contributes to RIPK3-mediated necroptosis, which in turn results in extracutaneous inflammatory pathologies [8]. Sharpin ${ }^{\text {cpdm }}$ mice lacking TNFR1 do not develop skin inflammation or systemic inflammation [8]. However, despite being rescued from dermatitis, Sharpin ${ }^{\mathrm{cpdm}} M y d 88^{-/-}$mice were only partially protected from immune cell dysregulation, suggesting that MyD88 does not regulate cell death in nonepidermal cells of Sharpin ${ }^{\mathrm{cpdm}}$ mice. Besides TNF, type I interferon signaling is required for sustained RIP3 activation and necroptosis in macrophages [20]. Importantly, cells lacking MyD88 undergo necroptosis in response to TNF or 
a

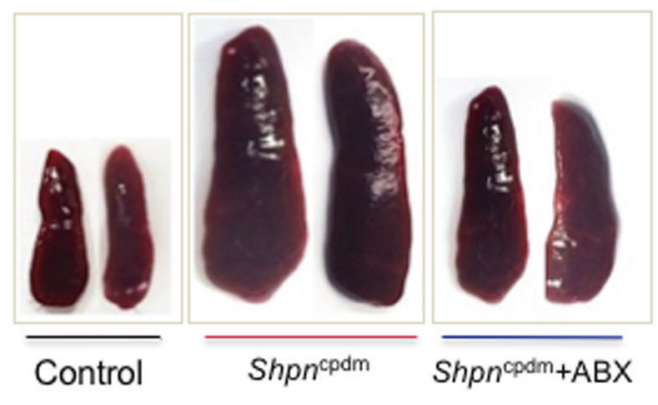

d
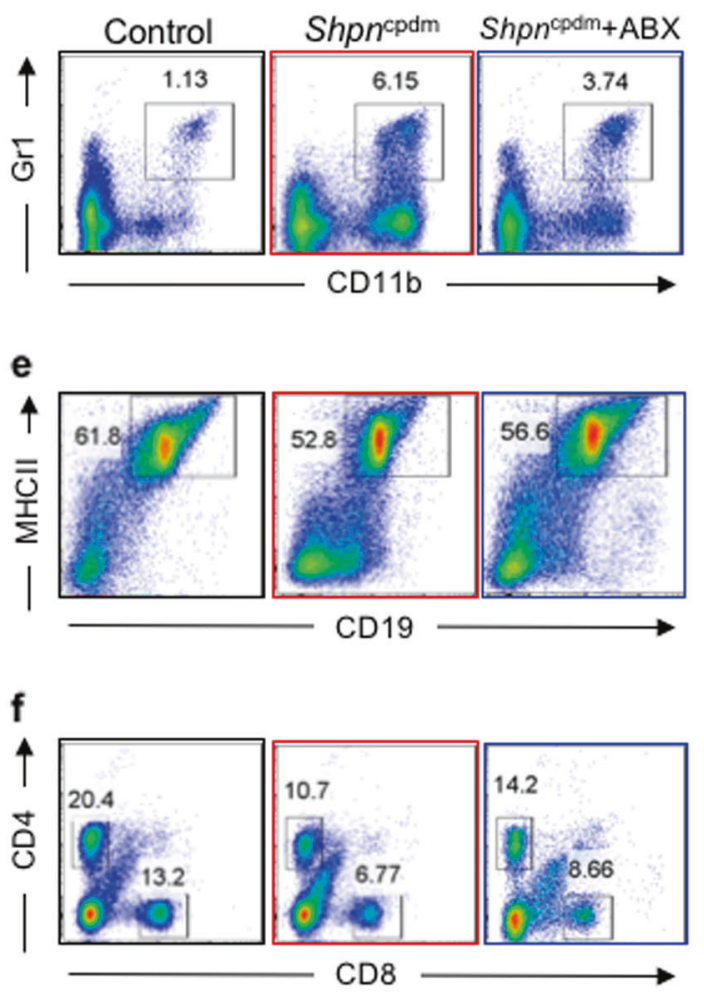

Fig. 5 Microbiota modulation partially prevents systemic inflammation and immune cell dysregulation in Sharpin ${ }^{\text {cpdm }}$ mice. a Representative image of spleens of control, Sharpin ${ }^{\mathrm{cpdm}}$, and Sharpin ${ }^{\mathrm{cpdm}}+$ $\mathrm{ABX}$ mice on post-natal day 60 . b, c Spleen weight (b) and splenocytes present (c) in control, Sharpin ${ }^{\text {cpdm }}$, and Sharpin ${ }^{\text {cpdm }}+$ ABX mice on post-natal day 60 . d-f Flow cytometry analysis of splenocytes from control, Sharpin ${ }^{\text {cpdm }}$, and Sharpin ${ }^{\text {cpdm }}+\mathrm{ABX}$ mice on post-natal day 60. Representative flow cytometry plots of $\mathrm{CD} 11 \mathrm{~b}^{+} \mathrm{Gr} 1^{+}$neutrophils

IFN, suggesting that MyD88 is dispensable for promoting necroptosis [20], which might explain the partial protection against extracutaneous inflammatory pathologies observed in Sharpin ${ }^{\mathrm{cpdm}} M y d 88^{-1-}$ mice. It would be interesting to see b

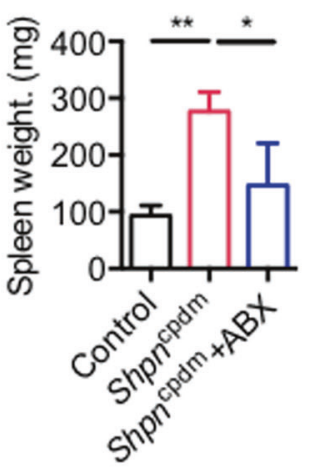

g

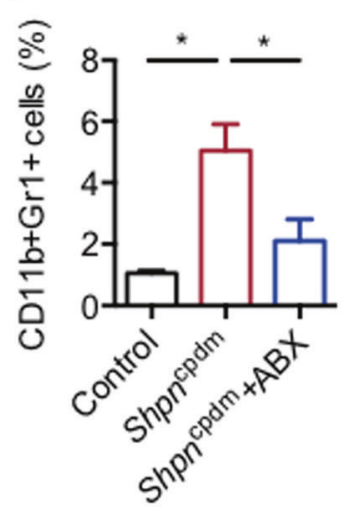

i

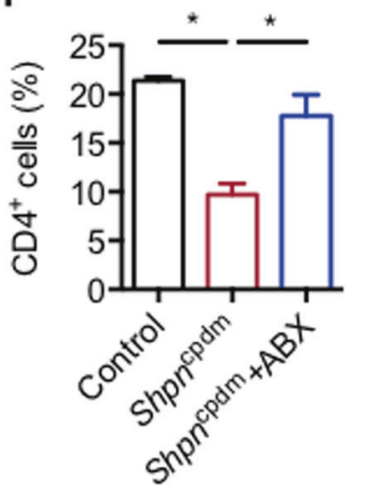

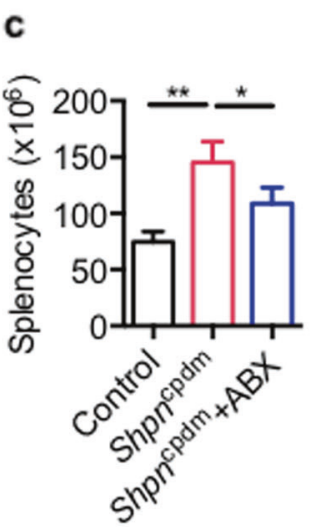

h
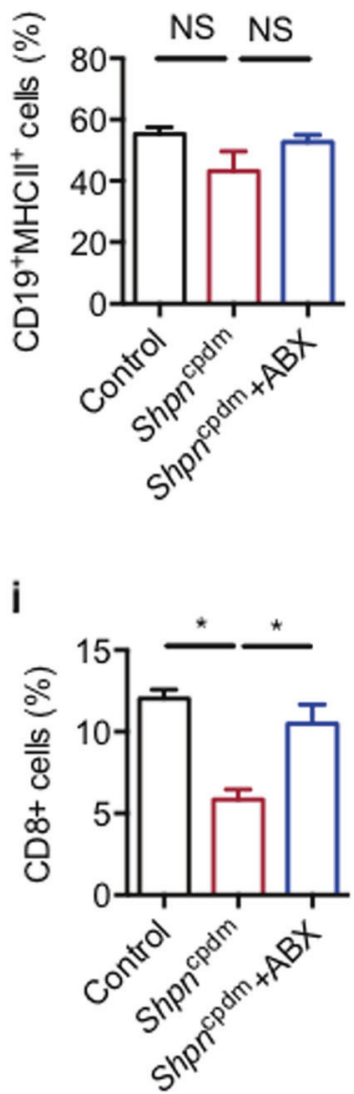

(d), $\mathrm{CD} 19^{+} \mathrm{MHCII}^{+} \mathrm{B}$ cells $(\mathbf{e})$, and $\mathrm{CD}^{+}$and $\mathrm{CD} 8^{+} \mathrm{T}$ cells $(\mathbf{f})$ cells in the spleen. $\mathbf{g}-\mathbf{j}$ Cumulative bar charts representing the frequencies of $\mathrm{CD} 11 \mathrm{~b}^{+} \mathrm{Gr}^{+}$neutrophils (g), $\mathrm{CD} 19^{+} \mathrm{MHCII}^{+} \mathrm{B}$ cells $(\mathbf{h}), \mathrm{CD}^{+}$ $\mathrm{T}$ cells $(\mathbf{i})$, and $\mathrm{CD} 8^{+} \mathrm{T}$ cells $(\mathbf{j})$ in the spleen. $S h p n^{\mathrm{cpdm}}$ corresponds to Sharpin $^{\text {cpdm }}$ mice. In the bar charts, data are presented as the mean \pm SEM and are representative of at least two independent experiments. $n=5-7$ for each group. $P$-values less than 0.05 are considered statistically significant. $* P<0.05, * * P<0.01$

whether the deletion of IFN signaling components protects Sharpin $^{\mathrm{cpdm}}$ mice from dermatitis and immune cell dysregulation. Moreover, the contribution of other innate immune sensors that might contribute to disease 


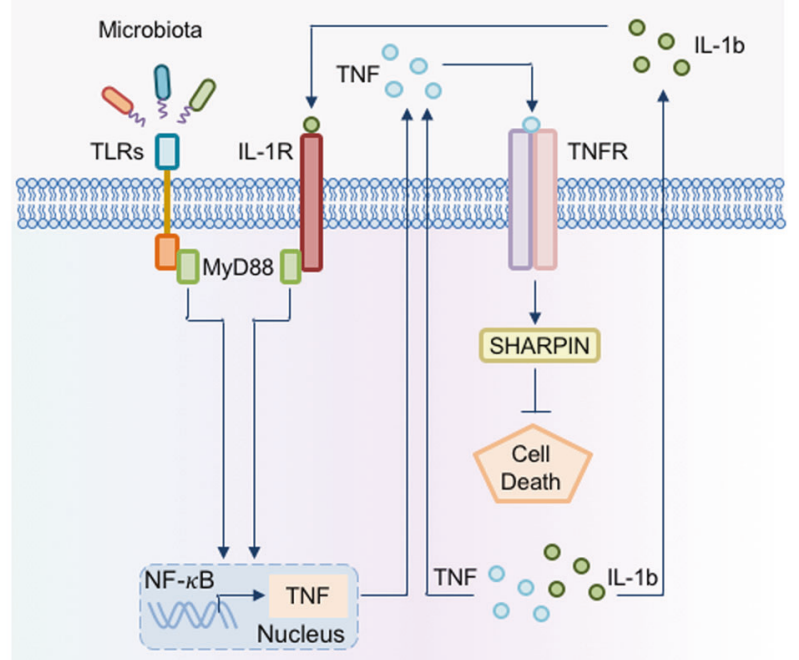

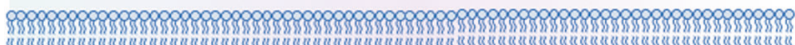
헤

Fig. 6 Model of how the innate immune adaptor MyD88 promotes skin inflammation in SHARPIN-deficient mice. MyD88, an innate immune adaptor molecule downstream of both TLRs and IL-1R, leads to the production of the inflammatory cytokine TNF. TNF then binds to TNF receptor for pro-survival cell signaling. However, in the absence of SHARPIN, aberrant TNF signaling leads to cell death, producing the inflammatory cytokines TNF and Illb

pathogenesis via TNF production still needs to be investigated. Microbial dysbiosis promotes several inflammatory diseases [21], and TLRs sense microbes via MyD88 [22]. The depletion of the microbiota partially protected against skin inflammation and immune cell dysregulation in Shar$\operatorname{pin}^{\mathrm{cpdm}}$ mice, suggesting that the microbiota acts as a trigger for both skin inflammation and extracutaneous inflammatory pathology. Overall, our findings suggest that MyD88 promotes the development of inflammatory skin disease in Sharpin ${ }^{\mathrm{cpdm}}$ mice and that neutralizing TNF or modulating the microbiota may attenuate many of the clinical symptoms of patients suffering from autoinflammatory skin diseases due to mutations in the LUBAC complex.

\section{Methods}

\section{Mice and breeding}

C57BL/6 and Sharpin ${ }^{\text {cpdm }}$ (stock no. 007599) mice were purchased from The Jackson Laboratory and bred at St. Jude Children's Research Hospital in a specific pathogen-free animal care facility. Myd $88^{-/}$mice have been described previously [23] and were bred with Sharpin $^{\text {cpdm }}$ mice to generate Sharpin ${ }^{\mathrm{cpdm}} M y d 88^{-/}$mice. The control animals that appear in all the figures included both C57BL/6 WT and Sharpin ${ }^{\mathrm{cpdm} /+}$ mice. Sharpin ${ }^{\mathrm{cpdm} /+}$ mice are completely normal and do not develop dermatitis or inflammatory disease. The animal studies were conducted under protocols approved by the St. Jude Children's Research Hospital Institutional Animal Care and Use Committee.

\section{Scoring of mice for dermatitis for disease-free curves}

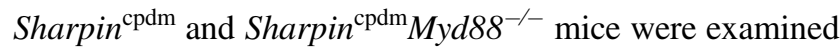
every week for signs of skin inflammation. These mice were scored as diseased on the day on which the first sign of dermatitis was observed.

\section{Cytokine multiplex assay}

Cytokines in the skin lysates were measured using multiplex ELISAs (MCYTOMAG-70K, Millipore). Skin lysates were prepared by homogenizing skin samples in ice-cold RIPA buffer with a homogenizer. A BCA Protein Assay Kit (Thermo Fisher Scientific) was used to normalize protein levels. Values below the reference range were assigned the lowest standard value.

\section{Spleen and PBL processing, fluorescent antibody staining, and flow cytometry analysis}

Spleens were ground using a 3-mL syringe plunger and passed through a $40-\mu \mathrm{m}$ filter to produce a single-cell suspension. Splenocytes were then treated with $2 \mathrm{~mL}$ of ammonium-chloride-potassium (ACK) lysis buffer to lyse red blood cells (RBCs). After lysis, spleen cells were resuspended in FACS buffer (phosphate buffered saline + $0.01 \%$ sodium azide $+5 \%$ fetal bovine serum) and stained with appropriate flow cytometry antibodies. Blood was collected by ventricular puncture, $50 \mu \mathrm{L}$ of each sample was transferred to a 96-well plate, and ACK lysis buffer was added for $5 \mathrm{~min}$ to lyse the RBCs. After centrifugation at $1500 \mathrm{rpm}$ for $5 \mathrm{~min}$, the lysis buffer step was repeated to completely lyse the RBCs. Then, the PBLs were stained with flow cytometry antibodies. RBC-free, single-cell suspensions of splenocytes or PBLs were stained with monoclonal antibodies against CD11b (2D7), MHCII (M5/ 114.15.2), CD4 (RM4-5), Gr1 (RB6-8C5), CD44 (IM7), CD19 (6D5), CD8 (53-6.7), and CD11b (M1/70) as previously described [9]. Fluorescently labeled cells were then analyzed using a FACS Calibur flow cytometer (BD Biosciences) and FlowJo software.

\section{Immunoblot analysis}

Proteins were extracted from skin samples or from fibroblasts by using RIPA lysis buffer supplemented with 
protease (11697498001, Roche) and phosphatase inhibitors (04906837001, Roche) as described previously [24]. Samples were resolved by $8-12 \%$ SDS-PAGE and transferred to polyvinylidene difluoride (PVDF) membranes (IPVH00010, Millipore). Blocking was performed in 5\% milk for $1 \mathrm{~h}$, after which the membranes were incubated with primary antibodies overnight at $4{ }^{\circ} \mathrm{C}$. The membranes were then incubated with HRP-conjugated secondary antibody for $1 \mathrm{~h}$, and proteins were visualized using SuperSignal Femto substrate (34096, Thermo Fisher Scientific). The primary antibodies used were anti-caspase-3 (bs0081R, Bioss; 1:1000), anti-cleaved caspase-3 (9661L, Cell Signaling Technology; 1:1000), anti-caspase-7 (9492, Cell Signaling Technology; 1:1000), anti-cleaved caspase-7 (9491, Cell Signaling Technology; 1:1000), and anticleaved caspase-8 (9746, Cell Signaling Technology; 1:1000).

\section{Real-time (RT-PCR) analysis}

RNA was extracted from $1.5 \times 1.5-\mathrm{cm}$ shaved skin sections by homogenization in TRIzol reagent (15596026, Thermo Fisher Scientific) followed by chloroform extraction and isopropanol precipitation. The extracted RNA was reversetranscribed into cDNA by using a High-Capacity cDNA Reverse Transcription Kit (4368814, Applied Biosystems). Real-time quantitative PCR was performed on an ABI 7500 RT-PCR instrument, using $2 \times$ SYBR Green (4368706, Applied Biosystems) and the appropriate primers, in accordance with the manufacturer's instructions. The sequences of the quantitative RT-PCR primers (forward/ reverse) were as follows: Tnf: 5'-CATCTTCT CAAAATTCGAGTGACAA-3', 5'-TGGGAGTAGACAA GGTACAACCC-3'; $\beta$-actin: 5'-GGCTGTATTCCCCTC CATCG-3', 5'-CCAGTTGGTAACAATGCCATGT-3'.

\section{Images and histopathology}

Mouse images were taken with a Canon digital camera. Formalin-preserved skin sections were processed and embedded in paraffin according to standard procedures. Skin sections ( $5 \mu \mathrm{M}$ thickness) were stained with hematoxylin and eosin $(\mathrm{H} \& \mathrm{E})$, and images were acquired using a Nikon wide-field light microscope.

\section{Statistical analysis}

All data are represented as the mean \pm SEM, and all experiments were repeated twice before being reported. Survival curves were analyzed using the log-rank (MantelCox) test. GraphPad Prism 6.0 software was used for data analysis. One-way ANOVA (with multiple comparison tests) followed by Dunnet's post test for more than two groups was used to compare the statistical significance between two groups. $* P<0.05, * * P<0.01$, *** $P<0.001$, $* * * * P<0.0001$.

Acknowledgements We thank A. Burton and N. Lantz for technical support, and members of the Kanneganti laboratory for their comments and suggestions. We also thank Keith A. Layock, PhD, ELS (Department of Scientific Editing, St. Jude Children's Research Hospital, Memphis, TN) for assistance with editing the article. This work was supported by grants from the National Institutes of Health (AI101935, AI124346, AR056296, and CA163507) and ALSAC (to T-DK).

\section{Compliance with ethical standards}

Conflict of interest The authors declare that they have no conflict of interest.

\section{References}

1. HogenEsch H, Gijbels MJ, Offerman E, van Hooft J, van Bekkum DW, Zurcher C. A spontaneous mutation characterized by chronic proliferative dermatitis in C57BL mice. Am J Pathol. 1993;143:972-82.

2. Seymour RE, Hasham MG, Cox GA, Shultz LD, Hogenesch H, Roopenian DC, et al. Spontaneous mutations in the mouse Sharpin gene result in multiorgan inflammation, immune system dysregulation and dermatitis. Genes Immun. 2007;8:416-21.

3. Gurung P, Kanneganti TD. Autoinflammatory skin disorders: the inflammasomme in focus. Trends Mol Med. 2016;22:545-64.

4. Tokunaga F, Nakagawa T, Nakahara M, Saeki Y, Taniguchi M, Sakata S, et al. SHARPIN is a component of the NF-kB-activating linear ubiquitin chain assembly complex. Nature. 2011; 471:633-6.

5. Ikeda F, Deribe YL, Skånland SS, Stieglitz B, Grabbe C, FranzWachtel $\mathrm{M}$, et al. SHARPIN forms a linear ubiquitin ligase complex regulating $\mathrm{NF}-\mathrm{\kappa B}$ activity and apoptosis. Nature. 2011;471:637-41.

6. Gerlach B, Cordier SM, Schmukle AC, Emmerich CH, Rieser E, Haas TL, et al. Linear ubiquitination prevents inflammation and regulates immune signalling. Nature. 2011;471:591-6.

7. Rickard JA, Anderton H, Etemadi N, Nachbur U, Darding M, Peltzer N, et al. TNFR1-dependent cell death drives inflammation in Sharpin-deficient mice. Elife. 2014; 3 https://doi.org/10.7554/ eLife.03464.

8. Kumari S, Redouane Y, Lopez-Mosqueda J, Shiraishi R, Romanowska M, Lutzmayer S, et al. Sharpin prevents skin inflammation by inhibiting TNFR1-induced keratinocyte apoptosis. Elife. 2014; 3 https://doi.org/10.7554/eLife.03422.

9. Gurung P, Sharma BR, Kanneganti TD. Distinct role of IL-1 $\beta$ in instigating disease in Sharpin ${ }^{\mathrm{cpdm}}$ mice. Sci Rep. 2016;6:36634.

10. Kawai T, Akira S. The role of pattern-recognition receptors in innate immunity: update on Toll-like receptors. Nat Immunol. 2010;11:373-84.

11. Kawai T, Akira S. Toll-like receptor downstream signaling. Arthritis Res Ther. 2005;7:12-19.

12. Lin $\mathrm{X}$, Kong $\mathrm{J}$, Wu Q, Yang Y, Ji P. Effect of TLR4/ MyD88 signaling pathway on expression of IL- $1 \beta$ and TNF- $\alpha$ in synovial fibroblasts from temporomandibular joint exposed to lipopolysaccharide. Mediat Inflamm. 2015;2015:329405.

13. Cohen P. The TLR and IL-1 signalling network at a glance. J Cell Sci. 2014;127(Pt 11):2383-90.

14. Akira S, Takeda K. Toll-like receptor signalling. Nat Rev Immunol. 2004;4:499-511. 
15. Douglas T, Champagne C, Morizot A, Lapointe JM, Saleh M. The inflammatory caspases- 1 and -11 mediate the pathogenesis of dermatitis in Sharpin-deficient mice. J Immunol. 2015; 195:2365-73.

16. Deguine J, Barton GM. MyD88: a central player in innate immune signaling. F1000Prime Rep. 2014;6:97.

17. Pandey S, Kawai T, Akira S. Microbial sensing by Toll-like receptors and intracellular nucleic acid sensors. Cold Spring Harb Perspect Biol. 2014;7:a016246.

18. Tumanov AV, Grivennikov SI, Kruglov AA, Shebzukhov YV, Koroleva EP, Piao Y, et al. Cellular source and molecular form of TNF specify its distinct functions in organization of secondary lymphoid organs. Blood. 2010;116:3456-64.

19. Segueni N, Benmerzoug S, Rose S, Gauthier A, Bourigault ML, Reverchon F, et al. Innate myeloid cell TNFR1 mediates first line defence against primary Mycobacterium tuberculosis infection. Sci Rep. 2016;6:22454.
20. McComb S, Cessford E, Alturki NA, Joseph J, Shutinoski B, Startek JB, et al. Type-I interferon signaling through ISGF3 complex is required for sustained Rip3 activation and necroptosis in macrophages. Proc Natl Acad Sci USA. 2014;111:E3206-13.

21. Koh JH, Kim WU. Dysregulation of gut microbiota and chronic inflammatory disease: from epithelial defense to host immunity. Exp Mol Med. 2017;49:e337.

22. Takeda K, Akira S. Microbial recognition by Toll-like receptors. J Dermatol Sci. 2004;34:73-82.

23. Hoshino K, Takeuchi O, Kawai T, Sanjo H, Ogawa T, Takeda Y, et al. Cutting edge: Toll-like receptor 4 (TLR4)-deficient mice are hyporesponsive to lipopolysaccharide: evidence for TLR4 as the Lps gene product. J Immunol. 1999;162:3749-52.

24. Karki R, Man SM, Malireddi RK, Kesavardhana S, Zhu Q, Burton AR, et al. NLRC3 is an inhibitory sensor of PI3K-mTOR pathways in cancer. Nature. 2016;540:583-7. 